Revue d'histoire des chemins de fer

\title{
Célestin Montcocol, 1879-1981 - Notice biographique
}

Jean-Pierre Jougla

\section{CpenEdition}

\section{Journals}

Édition électronique

URL : https://journals.openedition.org/rhcf/1889

DOI : 10.4000/rhcf.1889

\section{Éditeur}

Rails \& histoire

\section{Édition imprimée}

Date de publication : 1 juin 2003

Pagination : 106-113

ISBN : 0996-9403

ISSN : 0996-9403

\section{Référence électronique}

Jean-Pierre Jougla, «Célestin Montcocol, 1879-1981 - Notice biographique », Revue d'histoire des chemins de fer [En ligne], 27 | 2003, mis en ligne le 14 janvier 2015, consulté le 22 avril 2022. URL http://journals.openedition.org/rhcf/1889; DOI : https://doi.org/10.4000/rhcf.1889 


\section{Jean-Pierre JOUGLA}

\section{Célestin Montcocol, 1879-1981 \\ Notice biographique}

Le nom de Montcocol évoque à la fois la figure du grand entrepreneur disparu en 1981 et l'entreprise de travaux souterrains et de voies ferrées qui a participé jusqu'à ce jour à toutes les étapes du développement des réseaux souterrains de Paris.

On ne peut séparer les deux ; en 1900, année de la mise en service de la première ligne du métro, l'ingénieur débutant Célestin Montcocol fait partie de l'équipe de Fulgence Bienvenüe ; l'entreprise qu'il crée quelques années plus tard est restée familiale jusqu'à une date récente et, lorsqu'elle ne l'a plus été, ses nouveaux propriétaires ont pris soin de conserver son nom.

Une telle longévité n'est pas fréquente pour les entreprises dans cette profession ; elle rappelle celle de son fondateur, dont elle illustre la stature et les capacités d'adaptation: Célestin Montcocol est mort à plus de 102 ans ; il a été l'acteur ou le témoin de tous les progrès techniques survenus depuis le début de ce siècle dans la construction des souterrains.

Il naît le 26 juin 1879 en Avignon, dans la famille d'un petit entrepreneur en maçonnerie dont il est le second des deux fils ; ses parents le mettent en pension très jeune au collège et, au terme de ses études en 1898, il obtient son diplôme d'ingénieur de l'École nationale des arts et métiers d'Aix-en-Provence ; il débute à la Compagnie du métropolitain de Paris, la CMP, constituée en 1898 pour la construction des trois premières lignes projetées et devenues les lignes $n^{\circ} 1,2$ et 6 actuelles.

Deux mille ouvriers s'activent alors entre la porte Maillot et la porte de Vincennes à la construction de la première ligne, qui sera achevée en vingt mois, mais malgré tout en-dehors des délais prévus par suite des atermoiements des décideurs et qui sera inaugurée discrètement le 19 juillet 1900.

À la CMP, Célestin Montcocol rencontre Victor Labour qui a fondé en 1879 son entreprise de travaux publics, a réalisé de nombreux ouvrages d'art dans le Centre de la France, s'est maintenant installé à Boulogne-sur-Seine et exécute pour le métro des ouvrages de ventilation et d'accès ; c'est ce même Victor Labour qui déposera, en 1905, un brevet d'utilisation du carborandum dans les marches d'escalier du Métro, procédé qu'ont adopté pendant de nombreuses années la CMP puis la RATP. 
En 1905, il épouse la seconde fille de Victor Labour, Emma (alors que son frère aîné épouse la première) ; deux filles vont naître de cette union. Il ne tarde pas à quitter la CMP pour entrer dans l'entreprise de son beau-père qui a de son côté décelé ses capacités ; il s'associe avec lui en 1908 pour fonder l'entreprise en nom collectif Victor Labour et Célestin Montcocol.

Celle-ci obtient une médaille d'or à l'Exposition internationale de Milan en 1906, puis la grand prix de l'Exposition internationale de Londres en 1908 où Célestin Montcocol remporte en outre une médaille d'or à titre personnel.

Il en devient le gérant en 1910, puis le propriétaire au décès de son beau-père en 1912 ; il en fait une société anonyme qui porte désormais son seul nom.

En 1914, quatre-vingt dix kilomètres de lignes de métro sont déjà en exploitation ; le succès auprès du public et la fréquentation croissante du réseau ont eu raison des palinodies politiques ; des difficultés techniques considérables ont été résolues (la plus célèbre est la traversée sous-fluviale de l'actuelle ligne $\mathrm{n}^{\circ} 4$, où fut mise en œuvre pour la première fois une congélation du terrain) et un nouveau savoir-faire a été créé ; tout cela est dû à Fulgence Bienvenüe, dont Célestin Montcocol admira toute sa vie le talent et le caractère.

La guerre met tous les chantiers en sommeil ; Célestin Montcocol est affecté à Epernay comme officier dans le train des équipages ; lors de sa démobilisation, il est devenu responsable du Service automobile des télécommunications.

En 1919, Célestin Montcocol a quarante ans ; c'est un silencieux, peu expansif, aux colères rares mais violentes, homme d'autorité au caractère volontiers possessif mais dont les collaborateurs comme les proches admirent et craignent les jugements pénétrants, la mémoire infaillible et les capacités supérieures d'observation et d'anticipation.

Plus tard, ses petits-enfants le surnommeront «le vieux renard» ; il leur racontera de son côté que, à l'âge de sept ans, il se fit couper les cheveux par le coiffeur de Victor Hugo...

Sa curiosité est toujours en éveil et le restera jusqu'au terme de sa vie : en 1969, par exemple, âgé de quatre-vingt dix ans, il veille pour regarder en direct à la télévision la marche sur la lune.

Il sait toujours tout des gens qui ont affaire à lui ; il croit aux hommes, pas aux papiers et il aime à répéter à ses collaborateurs que l'argent se gagne sur les chantiers et non à la Bourse ; il n'y place pas le sien et il préfère les biens fonciers ou immobiliers qu'il répugnera toujours 
à revendre ; en 1933, par exemple, il achète dans le désert varois de Sainte-Maxime, lors d'une vente à la bougie, cent soixante-dix hectares de garrigues boisées et incultes qui brûlent périodiquement; près de cinquante années plus tard et après sa mort, c'est son petit-fils qui achèvera la réalisation du domaine hôtelier et du golf que Célestin Montcocol voulait y ériger en bordure de l'avenue qui porte maintenant son nom.

La période qui s'ouvre en 1919 voit les travaux reprendre à un rythme élevé et l'organisation des transports parisiens changer plusieurs fois ; le réseau de métro connait, jusqu'en 1939, un développement rapide auquel l'entreprise Montcocol, qui s'est installée quai de la Rapée à proximité du siège de la Compagnie du Métro, va prendre une part croissante sous l'impulsion de son fondateur pour devenir une des premières entreprises spécialistes du souterrain parisien.

Dans l'entre-deux-guerres, de 1921 à 1939, les travaux du métro de Paris représentent en valeur 3,4\% de l'ensemble des marchés publics de travaux pour toute la France, Génie militaire inclus; ce pourcentage atteint 8,5\% dans les années 1926-1931; relativement aux seuls travaux souterrains, les chiffres ci-dessus atteignent 48 et $70 \%$.

On a comparé le marché du métro de Paris à cette époque à un monopsone contrarié, où un donneur d'ordre unique, la Ville de Paris, a été confronté à un petit nombre de concurrents et s'y est adapté par un fractionnement approprié des lots : en réalité, la nécessité d’opérer dans des terrains fragiles et souvent aquifères, à proximité immédiate d'habitations et à partir d'emprises exiguës, faisait de la construction de grands souterrains dans Paris une spécialité, voire une exclusivité dans laquelle, en l'absence des moyens modernes de terrassement, de soutènement et de consolidation des sols, excellait le savoir-faire de quelques entreprises moyennes.

En fait trois entreprises : Desplats et Lefèvre, Les Établissements Billiard et Montcocol vont ensemble capter les deux tiers des commandes entre 1920 et 1936 et jusqu'à $83 \%$ dans la période 1920-1929 ; l'activité ne se ralentit que de 1922 à 1925, peut-être par suite de péripéties dans le processus de décision; les entrepreneurs cherchèrent alors du travail dans les travaux routiers; Célestin Montcocol créa dans ce but une filiale, La Route Rapide, qui fut un échec.

Au début de cette période qui couvre l'entre-deux-guerres l'entreprise fondée par Edmond Billiard en 1919 est la plus importante ; à partir de 1929, la Ville de Paris élargit ses appels d'offres à des grandes entreprises nationales et surtout à d'autres entreprises parisiennes, telles que Darras et Jouanin, Dumesny et Chapelle, Moinon, Lefrançois, etc. 
Mais Montcocol sera la seule à augmenter régulièrement son chiffre d'affaires, qui croît en moyenne de $10 \%$ par an de 1920 à 1925 et de $20 \%$ de 1925 à 1935 ; dans la période 1936-1939, les travaux effectués pour le métro et la Ville de Paris en représentèrent ensemble $60 \%$, soit $40 \%$ et $20 \%$ respectivement.

Dans les références de l'entreprise Montcocol pendant ces vingt années figurent toutes les catégories d'ouvrages liés à la construction du réseau de métro: tunnels, stations, accès, ouvrages techniques et de service, déviations d'égouts, bâtiments, etc., mais aussi des travaux effectués pour d'autres maîtres d'ouvrage publics ou privés et, notamment :

- les villes de Marseille, de Saint-Germain-en-Laye, d'Antibes (bâtiments et égouts) ;

- les chemins de fer: PO-Midi, PLM, puis SNCF;

- les ministères des Travaux publics, de la Guerre, de la Marine, de l'Éducation nationale pour lequel on note la construction de l'Institut du cancer à Villejuif, la nouvelle faculté de médecine, le muséum du Jardin des Plantes ;

L'activité de Célestin Montcocol dans cette période ne se borne pas à la direction de son entreprise ; il prend un rôle important dans l'organisation et les instances de la profession.

En 1927, il crée en collaboration avec des représentants ouvriers la Société de secours mutuel, qui comprend un bureau de placement et une caisse de secours ; cette même année, il fonde le Syndicat professionnel des entrepreneurs de souterrains de la Région parisienne.

En 1932, il est élu membre du comité de la Société des anciens élèves des Écoles nationales d'ingénieurs des arts et métiers en tant que délégué de la promotion 1895.

En 1936, il devient administrateur du Syndicat des entrepreneurs de travaux publics de France et participe à ce titre et en qualité de délégué à la négociation des accords Matignon.

En 1937, il fonde le Syndicat des entrepreneurs de travaux publics de la Région parisienne et en assume la vice-présidence ; il en deviendra le président en 1942.

En 1940, il participe à la fondation de la Fédération nationale des travaux publics dont il deviendra le vice-président en 1941 ; il sera nommé cette même année administrateur de la Caisse de cautionnement mutuel du bâtiment et de travaux publics. 
S'étant toujours intéressé à la formation, il entre à cette époque au Comité central de coordination de l'apprentissage et y est chargé d'étudier la création d'une école d'apprentissage pour les travaux publics ; sur sa proposition, sera créée en 1943 l’Ecole d'Égletons.

Le travaux du Métro commencent à décroître sensiblement dès 1936 ; pendant la guerre ils vont pratiquement s'arrêter et l'activité de l'entreprise, qui réussit à ne pas travailler pour l'occupant, se réduit considérablement.

En 1945, son chiffre d'affaires n'est plus que le quart de ce qu'il était; elle a gardé dans le métro l'activité d'entretien des voies et des ouvrages d'art, mais les grands travaux d'extension des réseaux ne reprendront qu'au début des années 1960 avec le lancement du RER.

Pendant ces premières années de l'après-guerre, Célestin Montcocol subit des épreuves personnelles dramatiques: ses deux gendres meurent jeunes, l'un en 1947, l'autre en 1954 ; il redevient, à plus de 70 ans, le chef des familles de ses deux filles et de ses six petits-enfants.

En 1958, son collaborateur immédiat Paul Magrou, ingénieur centralien qui est apparenté à sa famille et qu'il avait préparé à lui succéder, meurt également et quelques responsables quittent l'entreprise.

Pour remplacer Paul Magrou, il embauche en 1959 un autre centralien de 32 ans, Jacques Dubois, qui donne un nouveau départ à l'entreprise, confirmant sa réputation dans ses deux spécialités du souterrain et des voies ferrées.

Sous l'impulsion de Jacques Dubois et grâce aux équipes de jeunes cadres qu'il a su animer, l'entreprise renoue avec l'expansion : de 1958 à 1962, son chiffre d'affaires passe de 17,8 à 29,1 MF (valeur de l'époque, soit environ 70 millions d'euros en valeur actuelle) et son effectif de 1050 à 1270 personnes ; sur ces cinq années, les terrassements et fondations représentent en moyenne $35 \%$ de l'activité, les souterrains $37 \%$, les voies ferrées $16 \%$ et le bâtiment $12 \%$.

Pendant les années 1960 et 1970, ce chiffre d'affaires continue de croître en francs constants de $50 \%$ environ; entre 1965 et 1975, l'entreprise prend une dimension nationale comme le montrent ses principales références pendant cette période.

Dans la région parisienne, elle construit seule ou en participation diverses galeries : pour la Compagnie parisienne de chauffage urbain, pour le Service des eaux (et notamment une section de l'émissaire sud), pour les PTT, la traversée sous-fluviale particulièrement difficile de la galerie "Lutèce »; elle exécute pour la SNCF la gare souterraine de Paris-Austerlitz. 
Elle effectue sur la ligne $\mathrm{n}^{\circ} 11$ du métro la transformation des voies pour le roulement sur pneumatiques dont cette ligne a été le prototype; par la suite, au cours des années 1960 et 1970, la RATP développe cette technologie et lui confie la mise sur pneus des lignes $\mathrm{n}^{\circ} 1$, 4 et 6 ; ultérieurement, l'entreprise Montcocol prendra une part active dans la construction des voies du métro sur pneus de Marseille, puis du VAL de Lille.

En province, elle ouvre à Lyon une agence permanente qui assure un chiffre constant de l'ordre de 2 millions d'euros en valeur actuelle; elle obtient plusieurs marchés dans l'aménagement du canal de Provence ; elle participe aux travaux de remaniement des gares de Nantes, de Tours, d'Angers ; elle construit un collecteur à Wattreloo ; elle reprend et mène à bonne fin la construction très difficile du tunnel ferroviaire de Sainte-Dévote à Monaco ; en participation, elle exécute le renouvellement annuel de plusieurs centaines de kilomètres de voies à travers la France ; elle remet en état de nombreux tunnels de la SNCF, ou y intervient en urgence (lors de l'accident de Vierzy par exemple); elle se voit confier des lots importants par EDF dans l'aménagement d'Hermillon en Haute-Maurienne. Elle travaille également pour le Génie militaire, pour des sociétés d'autoroutes, pour l'aéroport de Roissy ; elle participe à la consolidation des carrières entreprise après l'effondrement de celle de Clamart en 1961, elle exécute une galerie souterraine pour l'Assemblée Nationale, etc. Elle exporte également son savoirfaire en participant à la construction des métros de Montréal, de Mexico, de Santiago du Chili et plus tard de Caracas.

Tout au long de l'ascension de son entreprise et essentiellement avant la guerre de 1939-1945, Célestin Montcocol a créé seul ou en participation des filiales dont l'ensemble constituait à la fin des années 1960 un groupe industriel important; on peut citer les Plâtrières de l'Est et Aubry-Pachot, une Société de nettoyage (La Moderne), une société de travaux ferroviaires (Landry frères), une entreprise de travaux électriques (la CELI), une société immobilière, une société hôtelière (la Résidence de Sainte-Maxime).

En 1974, il rachète l'Entreprise G. Verdier, spécialisée dans les galeries de montagne et il participe en 1977 (il a 98 ans...) à la création d'une société de stockage souterrain de fluides, la STOFLIS.

Parallèlement à cette ascension, il n'a cessé de jouer dans la profession un rôle de premier plan, par ses fonctions représentatives mais aussi du fait de son autorité personnelle ; pour de nombreux projets, eu égard à son expérience exceptionnelle du sous-sol parisien, on sollicite son avis. 
En 1948, il est nommé président du Centre de l'industrie française des travaux publics et le reste jusqu'en 1971.

En 1958, il est nommé administrateur de la Caisse nationale des entrepreneurs de travaux publics de France et le reste également jusqu'en 1971.

Sa curiosité et sa vitalité restent étonnantes : en 1969, à l'âge de 90 ans, il se rend sur le chantier d'un souterrain autoroutier à La Spezzia, à laquelle participe son entreprise, pour procéder au dernier tir de mine qui va relier les deux attaques.

Au début des années 1960, la construction de grandes infrastructures de transport reprend en Ile-de-France avec celle de la ligne A du RER et des voies autoroutières ; l'entreprise Montcocol participe à la réalisation de la quasi-totalité des lots de la ligne A du RER, dont certains, comme la construction sous les immeubles de la station Auber, présentaient des difficultés considérables.

Néanmoins, les temps ont changé ; la technologie et les moyens ont évolué ; les tunneliers commencent à apparaitre (le premier est mis en œuvre à Paris dès 1965, pour le percement du tunnel Étoile-Neuilly du RER) ; au front de taille, le mécanicien va supplanter progressivement le mineur-boiseur et au siège le financier prend peu à peu le pas sur le technicien.

Le savoir-faire et la capacité d'adaptation des entreprises locales traditionnelles ne suffisent plus ; elles doivent souvent se regrouper pour affronter la concurrence des grandes entreprises nationales qui disposent d'une surface financière très supérieure.

Dans ce nouveau contexte technique et économique, les fonds propres d'une entreprise restée fondamentalement familiale sont insuffisants et son développement implique objectivement qu'elle trouve des apports ou des appuis financiers substantiels ; de plus, au cours des années 1970, le flux des travaux issus des grands programmes de la décennie précédente se tarit rapidement.

Pour Célestin Montcocol, qui a toujours mené seul ses affaires, ces orientations sont difficiles à accepter et il ne les perçoit probablement pas comme absolument inéluctables ; il diffère la recherche d'un rapprochement et par deux fois renonce à des opportunités d'entrer en bourse en rachetant des entreprises cotées.

En 1977, malgré son âge, il suit toujours avec attention les affaires de son entreprise ; il y fait entrer pour lui succéder son petit-fils Thierry Ménétrel, qui s'est spécialisé hors du groupe dans la gestion financière. Afin de faire face à une conjoncture qui devient de plus en 
plus difficile, Thierry Ménétrel et Jacques Dubois vont s'efforcer de concentrer le moyens financiers sur les seuls travaux publics en cédant diverses filiales industrielles sans rapport avec cette activité ; par mesure d'économie, l'ensemble des surfaces que l'entreprise occupe dans Paris est revendu ; le siège et les dépôts sont transférés à Beauchamps dans le Val-d'Oise.

En 1978, Philippe Clément, président de la Fédération nationale de travaux publics dont Jacques Dubois est déjà à cette époque le trésorier, propose à ce dernier d'y prendre le poste de directeur général ; Célestin Montcocol, qui s'est investi toute sa vie dans les intérêts collectifs de la profession, ne tentera pas de s'y opposer malgré la perte que ce départ représente et Jacques Dubois quitte l'entreprise.

La conjoncture se dégrade, les résultats également et, en particulier, la trésorerie, faute de fonds propres suffisants; la situation de l'entreprise est de plus en plus difficile et ne pourra plus être redressée ; fin 1980, sous la pression des banques, Célestin Montcocol devra accepter, dans l'amertume, qu'elle soit rachetée par l'Urbaine de travaux, société du groupe de Jacques Genest.

Ce dernier la cède ultérieurement à COGIFER, filiale du groupe De Dietrich ; par la suite, COGIFER absorbe la branche voies ferrées et se sépare de l'activité travaux publics qui continue depuis sous le nom de Montcocol TP avec un autre propriétaire.

Une photo de famille de 1979 montre Célestin Montcocol, le jour de son centième anniversaire, levant un club pour frapper, sur sa pelouse, un swing qu'on devine encore vigoureux.

Il meurt le 27 décembre 1981 dans sa $103^{\mathrm{e}}$ année.

Il était chevalier de la légion d'honneur depuis 1934, officier depuis 1951, vice-président honoraire du Syndicat professionnel des entrepreneurs de travaux publics de France et d'outremer, président d'honneur du Syndicat professionnel des entrepreneurs de travaux publics de la Région parisienne et président d’honneur du Syndicat professionnel des entrepreneurs de travaux souterrains de France. 